\author{
Rino Cigui \\ Centro di ricerche storiche di Rovigno \\ Piazza Matteotti 13, HR-52210 Rovigno - Rovinj \\ cigui@crsrv.org
}

\title{
Alcuni aspetti della vita socio-economica a Umago nei secoli XVI e XVII
}

\author{
Prethodno priopćenje | Preliminary communication \\ UDK 314.143(497.5 Umag)«15/16« \\ Primljeno | Received: 14. II. 2013.
}

\begin{abstract}
Sintesi
L'autore del presente lavoro, utilizzando sia bibliografia nota sia fonti documentarie ancora inesplorate, si sofferma su quegli aspetti economico-sociali che furono di particolare rilevanza per la comunità di Umago nei secoli XVI e XVII, un'epoca contraddistinta da chiaroscuri in cui le premesse per il decollo socio-economico e demografico della località si scontrarono con il generale degrado ambientale e igienico-sanitario che, nonostante le notevoli risorse naturali del territorio e il trapianto di popolazioni, ebbe conseguenze deleterie sia sul piano della crescita economica che della popolazione.
\end{abstract}

\section{Izvadak}

Koristeći se poznatom bibliografijom i još neproučenim dokumentarnim izvorima, autor se u ovom radu zadržao na onim socijalno-ekonomskim aspektima koji su bili od presudnoga značaja za umašku zajednicu u XVI. i XVII. stoljeću, razdoblju prepoznatljivom po igrama svjetla i sjene, u kojem su se pretpostavke socijalnoekonomskoga i demografskoga uzleta mjesta suprotstavljale općem ambijentalnom i higijenskom urušavanju, a koje je, usprkos značajnim prirodnim rezervama područja i preseljavanju stanovništva, imalo pogubne posljedice i na gospodarski rast i na stanovništvo.

Parole chiave: Umago, economia, colonizzazione, condizioni igienico-sanitarie, XVIXVII secolo

Ključne riječi: Umag, gospodarstvo, kolonizacija, higijensko-sanitarne prilike, XVI. - XVII. stoljeće 


\section{Introduzione}

Lo studioso che volesse ricostruire un qualsiasi periodo della storia di Umago deve, per forza maggiore, fare i conti con la scarsità di fonti storiche documentarie dovuta alle ripetute distruzioni che hanno interessato gli archivi della cittadina. ${ }^{1}$ Accidentali o perpetrate per rappresaglia, tali distruzioni hanno significato la cancellazione della sua storia scritta, per cui oggi una qualsiasi indagine sul passato della località non può che risultare forzatamente sommaria.

Fatta questa doverosa premessa, diciamo subito che i secoli XVI e XVII furono cruciali per la storia di Umago. La località, che aveva vissuto nei primi secoli dopo il Mille un discreto periodo di prosperità economica e demografica che ne aveva decretato l'espansione sulla terraferma, nei secoli successivi fu inserita nel novero delle zone insalubri della provincia, e nonostante nel Cinque - Seicento vi fossero tutti i presupposti per un seppur modesto decollo economico e demografico, questo non avvenne a causa delle pessime condizioni igieniche e sanitarie che rallentarono la crescita della popolazione e, di riflesso, quella economica.

Il depauperamento demografico della città e del territorio, che metteva a rischio tutta l'attività agricola, fu contrastato dall'intensa azione colonizzatrice della Serenissima che vi trapiantò popolazioni provenienti dall'area balcanica e non solo nella speranza di ripopolare le campagne abbandonate e scongiurarne in tal modo il tracollo economico. Iniziava così quel lungo processo di insediamento di genti nuove, protrattosi per un secolo e mezzo, che se da un lato andava a colmare il vuoto demografico causato dalle frequenti epidemie e guerre, dall'altro alterava in maniera decisiva la struttura etnica del territorio.

\section{Umago nel XVI secolo: l'attività economica e il porto}

Nel 1540 il geografo isolano Pietro Coppo nell'opuscolo Del sito de Listria ci dava questa immagine di Umago e del suo agro: "Da salvori a Humago sono miglia cinque e mezzo del ditto spatio e un reduto over Villetta ditta Sipar a marina distante da Humago miglia tre Humago non ha antiquita alcuna fo edificato sopra uno scoglio quasi circunfluo da mar. Sopra la ponta de Humago non molto largo de tera sono secche soto acqua. El territorio e

1 Rino Cigui, "Contributo all'araldica di Umago", Atti del Centro di ricerche storiche di Rovigno (in seguito: ACRSR), XXIV, 1994, 241; idem, “Omago. Cenni storici di Pietro Kandler", ACRSR, XXXIV, 2004, 421. 
quasi tutto plano ferace da natura de Arbori silvestri et di biave lavorandolo bene, dalche fo nominata la terra. Ha dentro le secche reduto de Navilii grossi, ma per picholi un pocho de molo arente la terra, et quivi alquanto laria non e troppo buono, qual comenza esser infame dala ponta de Salvori et si extende per tutta la Riviera Marittima sino in Larsa et più et manco insalubre secondo la qualità et esser de luochi, et questo intravien per li tre venti nocivi Sirocho Ostro et Garbin, che batte tutta questa riva: et per esser tutta riva de piera vica grotosa sopra la qual repossano le acque si marine come piovane et se putrefano et li vapori tirati da razi solari alquanto in suso battuti da ditti flati nocivi infestano laria de quindi vien: che li lochi che assendono in qualche più altura non sono così cativi et morbosi. Da Humago a Citta Nova sono miglia XVII, et miglia doi distante si attrova San Pelegrin, inde a un miglio San Zuan dela Corneda, et de qui a San Lorenzo de Daila miglia uno”. ${ }^{2}$

Come si nota, il Coppo poneva l'accento sulle caratteristiche geomorfologiche e naturali della località ricordate da quasi tutti i corografi del XVI e XVII secolo, e che furono il suo tratto distintivo: l'origine insulare, la presenza del porto, la fertilità della terra e la presenza di boschi, le precarie condizioni sanitarie e il conseguente spopolamento.

Il porto di Umago, sorto nell'insenatura naturale della cittadina, risultava all'epoca sufficientemente spazioso e al riparo dai venti. Oltre ad essere il punto di riferimento per il commercio di tutto il territorio, la sua posizione geografica di fronte alla Serenissima ne faceva una tappa quasi obbligata per chi volesse raggiungere la nostra penisola o spingersi verso le isole quarnerine e la Dalmazia. ${ }^{3}$ Definito porto considerevole da Luca da Linda, notabile da Nicolò Manzuoli, ${ }^{5}$ buonissimo da Giacomo Filippo Tommasini ${ }^{6}$, poteva ricevere, stando alle parole di Prospero Petronio, "ogni Vascello dal quale alle volte faranno paranza o vella trenta o quaranta Legni”7 e, così Fortunato Olmo, "nel Verno vi si vederanno alle fiate più di cento vas-

2 Attilio Degrassi, "Di Pietro Coppo e delle sue opere, documenti inediti e l'opuscolo Del sito de Listria ristampato dall'edizione del 1540", Archeografo triestino (in seguito: $A T$ ), XI, 1924, 383. Sulla vita e le opere del Coppo si veda Autori Vari, Petrus Coppus fecit. De summa totius orbis, Pirano 2006, 7-24.

3 Egidio Ivetic, "La classe dirigente veneta e i piani di risanamento dell'Istria - ruoli e prospettive di sviluppo per Pola in un discorso del primo Seicento", ACRSR, XXII, 1992, 298. L'autore cita una relazione di Pietro Matteacci del 1625 nella quale si ricordano i porti di Cittanova, Umago, Pirano e Capodistria validi solo per i traffici commerciali e per la sosta prima di attraversare il golfo.

4 Luca da Linda, "Relazioni e descrizioni universali e particolari del mondo", AT, II, 1830, 93.

5 Nicolò Manzuoli, Nova descrittione della provincia dell'Istria, Venezia 1611, 34.

6 Giacomo Filippo Tommasini, Commentari storico geografici della provincia dell'Istria, Trieste 2005, 293.

7 Prospero Petronio, Delle memorie sacre e profane dell'Istria (in seguito: Delle memorie), Trieste 1968, 202. 
celli venuti da diverse parti". ${ }^{8}$ Per le imbarcazioni minori c'era un piccolo molo verso terra, il cui pontile, nel primo decennio del XVI secolo, venne restaurato grazie alla concessione veneziana di “ducati 10 all'anno del dazio dell'oglio per 5 anni”. ${ }^{9}$

Nel porto trovavano collocazione i cosiddetti fari, costituiti da cinque tronchi d'albero riuniti tra loro e fissati sul fondo, sui quali venivano legate le imbarcazioni. Vista la loro natura, si rendeva spesso necessaria la loro sostituzione: nel 1589, ad esempio, fu concesso alla comunità di Umago “di poter far tagliare nel bosco di Farnè, giurisdizione di Buie, 50 roveri per costruire i fari di quel porto, a sicurezza dei vascelli”. ${ }^{\circ}$ Sulle secche, un segnale in muratura dotato di fanale luminoso indicava l'ingresso del porto. Il 25 maggio 1669 il Senato veneto ingiungeva al podestà e capitano di Capodistria di far riparare il faro nel porto di Umago, ${ }^{11}$ ordine che tuttavia venne disatteso se nella relazione di Pietro Loredan (1670) leggiamo che "il Farro di quel Porto è cadente, et hora con puochiss.a spesa potrebbe aggiongersegli il riparo, nè in questo il publico ne risentirebbe un picolo, mentre quella Comunità coll'utili del fontico s'esibisce di farlo". ${ }^{12}$

Quanto fosse importante segnalare l'ingresso del porto è lo stesso Loredan a rilevarlo: "Di che benefitio sia il sostentamento d'esso Farro è molto ben noto alla publica sapienza; poichè essendo erretto in sito che dà a conoscere dove da una parte vi sono gli scagni formati dalla natura, et dall'altra l'antica purpurella fabbricata per la sicurezza d'essa terra, non c'è dubbio che venendo abbattuto non sarà chi arrischi presentarvisi se non in occasione che il tempo stesso gli prometta una felice navigazione; si che quelli che sfuggendo l'impeto delle burasche fossero per salvarsi in Porto precioso, non ardiranno d'accostarvisi per la certezza che non incontrando per appunto in quel stretto per il quale deve seguirne l'introduzione, di rimanervi sopra le secche colla perdita del Vascello e delle vite; ma s'esporanno più facilmente all'evidente rischio d'esser sommersi dall'onde”. ${ }^{13}$ Era quindi nell'interesse di tutti avere un porto efficiente e sicuro: per i veneziani, in

8 Fortunato Olmo, "Descrittione dell'Histria", Atti e memorie della Società istriana di archeologia e storia patria (in seguito: $A M S I$ ), I, 1885, 149-172.

9 "Senato Mare. Cose dell'Istria", AMSI, IX, 1894, 87.

10 "Senato Misti", AMSI, XII, 1896, 59.

11 "Senato Rettori, Deliberazioni Secrete di Senato - Serie Rettori”, AMSI, XIX, 1903, 29; Dario Alberi, Istria. Storia, arte, cultura, Trieste 1997, 1050.

12 "Relazioni dei podestà e Capitani di Capodistria", AMSI, VIII, 1892, 109.

13 Ibidem. 
quanto una contrazione dei traffici avrebbe fatto sì che "quella terra venghi maggiormente inabitata”; per i cittadini di Umago, che continuavano ad abitare la località "a riguardo del benefitio che conseguiscono dal Porto stesso per l'occasione che hanno dell'esito delle loro entrate et di qualche puoco traffico". ${ }^{14}$

Come si è potuto constatare, nel porto si concentrava il commercio di quasi tutto il territorio umaghese, quest'ultimo in prevalenza pianeggiante e circondato da amene colline, "ferace da natura de Arbori silvestri et biave" per il Coppo, "molto pingue di frumenti, vini, ogli, pascoli, boschi” nella descrizione che ne dava il Petronio ${ }^{15}$; nel suo poema "Histria", anche il vescovo triestino Andrea Rapicio lodò il vino dolce spumante prodotto nel territorio e la varietà di pesce del suo mare ${ }^{16}$. All'epoca i vigneti venivano coltivati $a$ piantada bassa, dalla caratteristica forma a cespuglio, e appena dal primo Seicento cominciarono a diffondersi quelli a piantada alta, diversi dai precedenti per il fusto più elevato ed i filari sostenuti da pali in modo da sfruttare meglio il terreno sottostante per la semina dei cereali. L'olivo invece, la cui coltivazione in Istria aveva un'antichissima tradizione, si diffuse nell'agro umaghese su vasta scala solo a partire dagli anni $1650-60 .{ }^{17}$

Tutta l'attività agricola, l'allevamento del bestiame, la pesca e il commercio del pesce erano regolati da precise disposizioni statutarie (Statuto del 1540). ${ }^{18}$ Tra tutti i possessori di vigne di una certa estensione che avessero superato i sedici anni di età venivano scelti, "quod singulo anno in festo sancti Michaelis de mense septembris", otto guardiani detti saltuari i quali eleggevano un gastaldo al quale, entro tre giorni, relazionavano su eventuali danni nei vigneti e nella campagna in generale affinché questi potesse presentare denuncia al podestà e ai giudici. Se il danno non veniva denunciato, i guardiani pagavano di tasca propria. Che quella dei saltuari fosse una carica tutt'altro che formale lo dimostra l'obbligo di rimanere nella frazione di territorio loro assegnata, senza allontanarsi mai, "in festo sancti Iacobi de mense julii usque quo reperientur uvae vindemiandae” pena il pagamento di quaranta soldi. ${ }^{19}$

\footnotetext{
14. Idem, 89.

15 Denis Visintin, “Cenni storici sulle vicende dell'agricoltura istriana”, Il comune di Umago e la sua gente, Trieste $1999,25$.

16 Bernardo Benussi, “Lo statuto del comune di Umago” (in seguito: “Lo statuto”), AMSI, VIII, $1892,235$.

17 Egidio Ivetic, L'Istria moderna. Un'introduzione ai secoli XVI-XVIII, Trieste-Rovigno 1999, 78.

18 Benussi, "Lo statuto", 227-313.

19 Idem, 303.
} 
I danneggiatori di oliveti, frutteti ed alberi in genere pagavano cinque lire di piccoli al comune e altrettanto al proprietario che aveva subito il danno; dovevano inoltre risarcire il danno "et hoc pro qualibet arbore". Era altresì severamente proibito asportare dagli orti altrui erba, frutta o legna: in questo caso la multa da pagare ammontava a tre lire venete più il risarcimento del danno al proprietario. Il vicino del contado o abitante di Umago che volesse arare extra districtum Humagi doveva versare al comune il terratico "perinde ac si cultivasset, aut cultivari fecisset in districtu Humagi in poena librarum decem parvorum". ${ }^{20} \mathrm{E}$ per far fronte alle ripetute carestie che colpivano il territorio, si proibiva l'esportazione di frumento, biade e legumi senza il consenso del podestà e dei giudici.

Una serie di normative, aventi quale obiettivo la tutela del prodotto locale, regolavano poi la produzione e la vendita di vino. A nessuno era permesso condurre vinum forense nella località e suo distretto "excepto ejus vino, quod conducere possit solum in una ante festum sancti Michaelis de mense septembris, jurando illud esse ex propriis suis vineis"; un mese di carcere e venticinque lire era la pena per i trasgressori. Anche la vendita di vino forestiero, fintanto che vi fosse la disponibilità di vino locale, era sanzionata con una penale di venticinque lire. ${ }^{21}$

Tra le disposizioni in materia di animali vi era quella che vietava tassativamente la presenza di maiali dentro le mura della terra, mentre era lecito tenerli legati nel borgo. Onde evitare che gli animali condotti all'abbeveraggio "per solitam stratam Spineti, Flandrae, et per capita vinearum Flandrae versus Rosaceum” danneggiassero i vigneti, questi dovevano essere circondati da siepi; solo in questo caso il proprietario delle bestie era responsabile del danno eventualmente arrecato. Restrizioni in tal senso riguardavano anche gli animali minuti (ovini e caprini) che "a montibus descenderint in hoc territorium, quae accesserint ad lacum causa potandi" ai quali non era permesso pascolare super Muglela né "a lama infra versus vineas", pena il pagamento di tre lire. ${ }^{22}$ Infine, a nessuno era permesso di cacciare nei vigneti "a dimidio martio usque ad festum sancti Michaelis de mense septembris sub pena soldorum centum parvorum pro quolibet et qualibet vice". Di giorno la caccia nei vigneti non doveva avvenire mai a cavallo o con reti; se però si cacciava di notte, la pena prevista era di "librarum

20 Idem, 297.

21 Idem, 298.

22 Idem, 302. 
decem, et perdendi retia; quilibet possit accusare, tenebitur de credentia, et habeat tercium dictae poenae". ${ }^{23}$

Due capitoli dello Statuto di Umago regolavano la pesca, la vendita e il prezzo del pesce. Ogni pescatore, fosse del luogo o straniero, era tenuto a pagare al comune una quota di "duodecim parvulis unum, in poena tertii, et librarum quinque”. Durante tutta la quaresima et vigiliarum jejunio, il pesce non doveva essere venduto in quantità inferiore a mezza libbra. In dicto tempore il prezzo delle angusigole (angusigulas) ammontava a due soldi la libbra, negli altri periodi dell'anno era "pro parvulis decem de octo pro libra”; le menole (menulas) venivano portate in pescheria e vendute "decem pro centenario, usque ad milliarum, et non ultra, habendo soldum unum pro singulis decem menulis". ${ }^{44}$

Lo sfruttamento dei boschi, di cui, come abbiamo potuto appurare, il territorio umaghese era ricco, rappresentava una voce importante dell'economia locale e non solo. I boschi, infatti, oltre a offrire legna da ardere alla popolazione, fornivano soprattutto legname da costruzione alla Serenissima che, sin dal 1476, aveva emanato uno dei più organici interventi normativi in materia di legislazione forestale. ${ }^{25}$ Tale regolamentazione era diretta a prevenire i danni causati ai boschi da persone e da animali, a scongiurare i pericoli di incendio, a promuoverne il rinnovamento e lo sfruttamento razionale con cicli di taglio quinquennali ed ottennali. ${ }^{26}$

A partire dal XVI secolo la Repubblica di Venezia ordinò la stesura dei catasti, le verifiche documentate dei boschi istriani, mediante i quali si aveva una visione completa delle dimensioni e, soprattutto, della resa preventiva di ogni singolo bosco. ${ }^{27}$ Il catasto del 1566, compilato dal Provveditore sopra legne in Histria et Dalmatia Fabio da Canal, fornisce un quadro preciso della consistenza forestale nel territorio di Umago. ${ }^{28}$ Nove erano i boschi comu-

23 Idem, 303.

24 Idem, 299

25 Emanuela Casti Moreschi - Elena Zolli, Boschi della Serenissima. Storia di un rapporto uomo - ambiente, Venezia 1988, 11. Cfr. Ivone Cacciavillani, Istria veneziana, Milano 2012, 115-118.

26 Vincenzo Morosini IV, Catastico generale dei boschi della provincia dell'Istria (1775 - 1776), Trieste 1980, (a cura di Vjekoslav Bratulić), p. V.

27 Ivan Pederin, “Il registro dei boschi dell'Istria occidentale del 1541-42”, ACRSR, XIV, 1983-1984, 153. Una lettera ducale del 2 aprile 1534 ordinava l'istituzione di registri di boschi per l'Istria, e nel 1538 veniva istituito l'Ufficio del Provveditore sopra le legne in Istria, Isole del Quarnero e Dalmazia. Il registro di Giammaria Spuazza del 1541-42 è il più vecchio della penisola e uno dei più antichi d'Europa.

28 Danilo Klen, "Katastik gorivog drva u istarskim šumama pod Venecijom (sastavljen od Fabija da Canal, god. 1566)" [Il catastico della legna da ardere nei boschi istriani durante il dominio veneto, compilato da Fabio da Canal, nel 1566], Vjesnik historïskog arhiva u Rijeci i Pazinu (in seguito: VHARP), XI-XII, 19661967, 25-29. 
nali: il Bosco Comun (due miglia di circonferenza) dal quale "si trazzerà doi mille stroppe di legne per esser bon fondi”; il bosco Val Castagna presso la Madonna del Carso (sei miglia di circonferenza) dal quale "si trazzerà sei mille stroppe di legne, et ogni quatro anni altrettante per essere bonissimo fondi”; il Bosco Pizzuda (tre miglia e mezzo di circonferenza) "al presente si farà stroppe III $^{c}$ di legne, ma diuidendosi i tagli in quatro parti, da esso bosco ogn'anno se ne tagliarà stroppe mille et più, et il taglio sarà continuo, perchè il fondo è buono et attissimo a produre nel termine sodetto"; il Bosco Martello (un miglio di circonferenza) "si farà al presente stroppe trenta di legne”; il Bosco Lama (un quarto di miglio di circonferenza) "hora si farà stroppe Cento insieme con altra sorte di legne oltra li detti cerri alti, et ogni quatro anni renderà Stroppe cento cinquanta”; il Bosco Grande "si farà stroppe Seimille, et più di legne"; il bosco detto Punta comunale di S. Lorenzo di Daila (due miglia e mezzo di circonferenza) "fra cingue anni doppo la prohibitione hora fatte si farà stroppe doimille di legne”; il Bosco Carobia (tre miglia di circonferenza) "fra termine delli anni cinque delle prohibitioni si farà stroppe doi mille cinquecento di legne, et così per l'auuenire"; il bosco Punta di Rosaz (due miglia di circonferenza) "fra anni cinque doppo li bandi hora fatti, si farà stroppe doimille di legne et così per l'auuenire”. Nel feudo di S. Giovanni della Cornetta dei signori Verzi era bandito il taglio per cinque anni, scaduti i quali, si sarebbero fatte duemila stroppe annue. Vi erano poi numerosi boschi di proprietà privata che fornivano però una quantità di legname decisamente inferiore.

Le grandi operazioni di abbattimento degli alberi ordinate dalla Repubblica venivano svolte dagli abitanti come carratada, ${ }^{29}$ un'imponente organizzazione che coinvolgeva uomini e mezzi e che aveva lo scopo di raccogliere e trasportare l'ingente legname dell'Istria. Il trasporto avveniva per mezzo di carri agricoli (carezi) verso uno scalo marittimo (carigador) dove si effettuava l'imbarco per Venezia; i carigadori nel territorio di Umago erano siti nel porto, a S. Giovanni della Cornetta (in località Carigador) e a S. Lorenzo di Daila. Il numero di carezi impiegati nel trasporto dipendeva dalla quantità di alberi tagliati: nel 1542, ad esempio, per il trasporto di 288 tronchi abbattuti nei boschi dell'Umaghese si stimarono necessari 400 carri, nonostante i buoi da traino a disposizione fossero solo 247. Nei decenni successivi, però, le autorità veneziane dovettero fare i conti con la drastica riduzione degli

29 Silvio Facchini, La grande carratada istriana, Trieste 1997, 45. 
animali da trasporto predisposti dalla terra di Umago e dagli altri comuni istriani per le necessità della Serenissima, riduzione che andava ricercata nella volontà della popolazione di sottrarsi all'onere della carratada con la vendita dei buoi o con il trasferimento dal territorio veneto in quello austriaco. Nel decennio 1542-1552 i buoi da lavoro registrati nella podesteria umaghese scesero di conseguenza da 247 a 153, per attestarsi a 123 nel 1560 (il 50\% in meno rispetto a vent'anni prima) e crollare a 34 nel $1660 .^{30}$

A prescindere dallo sfruttamento dei boschi da parte della Dominante, nei secoli XVI e XVII le discrete risorse naturali dell'agro umaghese crearono i presupposti per un seppur modesto decollo economico e demografico della località, che tuttavia non avvenne a causa delle pessime condizioni igienico-sanitarie che ne rallentarono la crescita demografica e, di riflesso, quella economica.

\section{Le condizioni igieniche e sanitarie}

La località, che aveva vissuto nei primi secoli dopo il Mille un discreto periodo di prosperità economica e demografica, testimoniata dall'espansione dell'abitato sulla terraferma, nei secoli XV - XVII fu soggetta a un profondo depauperamento demografico ed economico, ascrivibile alle guerre, alle cicliche incombenze epidemiche e alle carestie, che contribuì ad alimentarne la fama di luogo pestilenziale e malarico. Anche lo stato di indecenza in cui versava allora la città accelerò sicuramente il degrado della stessa, e a nulla servirono le disposizioni statutarie in materia di igiene pubblica che proibivano di tenere "porcos aut porcas in terra Humagi" e di gettare "aquam mundam aut immundam de alto in via Communis de die vel de nocte a balconis, fenestris, aut scalis". ${ }^{31}$ Tali disposizioni, che dovevano richiamare la popolazione all'osservanza di salutari regole di pulizia, si rivelarono però insufficienti e non arginarono le cattive abitudini igieniche, che pregiudicarono pertanto la salute pubblica trasformando la cittadina in un potenziale focolaio epidemico.

La documentazione in nostro possesso ci permette una ricostruzione alquanto sommaria delle fenomenologie morbose che hanno interessato la località. Per quanto concerne la peste, le prime notizie certe risalgono al

30 Danilo Klen, "Mletačka eksploatacija istarskih šuma i obvezan prevoz drveta do luke kao specifičan državni porez u Istri od 15. do kraja 18. stoljeća" ¿Lo sfruttamento veneziano dei boschi istriani e il trasporto della legna come imposta statale specifica in Istria dal XV alla fine del XVIII secolo], Problemi sjevernog Jadrana, I, 1963, 244-245.

31 Benussi, "Lo statuto", 296-297. Libro IV, cap. 35 e 39. 
1424 quando, nei mesi di giugno e luglio, il contagio infierì a tal punto che il podestà Jacopo Duodo ottenne, propter epidemiam sevientem, il permesso di "piantar per due mesi stanza fuori di quella terra”. ${ }^{32}$ Allo stesso fu concessa licenza di andare a Venezia per dieci giorni a curarsi dalle febbri, termine che slittò però sino al 15 di agosto perché malato "per unam panochiam". ${ }^{3}$ Dopo una breve tregua di tre anni, il morbo pestilenziale infierì nuovamente a Umago nel 1427. Proveniente a quanto sembra dal Veneto, invase dapprima Trieste, per estendersi successivamente lungo tutta la costa istriana da Salvore a Pola decimandone la popolazione. ${ }^{34}$

Nel 1507 la peste fece nuovamente la sua comparsa, portata questa volta dalle navi veneziane che facevano sosta nel porto. ${ }^{35}$ Oltre al flagello, la cittadina dovette sopportare pure una tremenda carestia che costrinse la popolazione a invocare il soccorso della Serenissima, la quale concesse immediatamente alla comunità "una dilazione di tempo per il pagamento del debito contratto... per 80 stara di farina: quindi l'assegno di ducati 10 all'anno del dazio dell'oglio per 5 anni da essa spesi nel ristauro del pontile del porto: inoltre l'immunità ed esenzione per 5 anni de ogni angaria real et personal: finalmente il permesso di estrar cinquecento stara di frumento da luoghi fuori di Venezia per venderli nel fondaco di Umago". ${ }^{66}$

Nei decenni successivi del XVI secolo, stando almeno alle fonti disponibili, non vi sarebbero state a Umago altre emergenze sanitarie connesse in qualche modo alla peste, anche se non possiamo escludere del tutto un coinvolgimento della località nelle cicliche incombenze epidemiche che contrassegnarono il periodo. Disastrose furono invece le conseguenze del contagio propagatosi nel 1630 per opera dei marinai veneziani che facevano scalo nel porto; le tristi condizioni e lo spopolamento della città, ridotta, secondo il Kandler, a poco più di una decina d'anime, si possono dedurre dal fatto che a sessant'anni di distanza, nel 1690, la località contasse appena 380 abitanti, una cifra che corrispondeva pressappoco alla metà della popolazione precedentemente attestata. ${ }^{37}$

32 "Senato Misti", AMSI, VI, 1890, 24. Anche in Bernardo Schiavuzzi, "Le epidemie di peste bubbonica in Istria. Memorie storiche raccolte da Bernardo Dott. Schiavuzzi”, Pagine Istriane, 11-12, 1912, 262-263.

33 "Senato Misti", AMSI, VI, 1890, 25.

34 Pietro Kandler, L'Istria, VI, 1851, 83.

35 Ernesto Fumis, Pagine di storia umaghese, Trieste 1920, 40.

36 "Senato Mare", AMSI, IX, 1893, 86-87.

37 Luigi Parentin, Incontri con l'Istria, la sua storia e la sua gente (in seguito: Incontri con l'Istria), vol. II, Trieste 1991, 23. Cfr. Sergio Cella, "Studi sull'Istria del '600", AMSI, LXIX, 1969, 10. 
$\mathrm{Fu}$ a causa del frequente imperversare delle pestilenze che il popolo umaghese coltivò speciale devozione a $\mathrm{S}$. Rocco, alla cui intercessione ricorse ad ogni manifestarsi del morbo funesto, in onore del quale fu costituita l'omonima confraternita (Scola S. Rochi). Per volontà della stessa, nel 1514 venne edificata una chiesetta votiva sita "extra oppidum et in Burgo Umagi”, consacrata l'anno seguente dal vescovo emoniense Marc'Antonio Foscarini in un clima molto teso a causa delle continue liti tra il vescovato tergestino e quello cittanovese per il possesso della parrocchia di Umago; ${ }^{38}$ all'interno, sono visibili ancora oggi le due pietre tombali in cui venivano sepolti i confratelli, che recano scolpiti in rilievo il caratteristico bordone e il cappello del Santo con la scritta: Confraternitas S. Rochi MDXCIIII T.D.M.B. ${ }^{39}$

Oltre la peste, Umago ebbe a soffrire particolarmente la malaria, che la trasformò da nobile oppidum ${ }^{40}$ in località malarica per eccellenza. Nei secoli XVI e XVII la cittadina venne inserita nel novero delle zone insalubri della provincia, comprendente tutta la costa da Salvore a Capo Promontore. Le testimonianze degli scrittori di cose istriane in tal senso sono numerose: nella sua opera corografica De situ Istriae (1540), il medico piranese Giovan Battista Goineo non mancò di sottolineare le meschine condizioni in cui versavano Umago e Cittanova "civitates ambae ob aeris intemperiem hand omnino tutae. Humacum tamen est portu, et clementiori coelo alteri praestat", ${ }^{41}$ mentre l'ispettore veneziano Giovanni Battista Giustiniano, che visitò la cittadina nel corso del viaggio che lo portò nel 1553 in Dalmazia, la definì "quasi inabitata e desolata”. ${ }^{42}$ Nel 1625 il provveditore veneto Fran-

38 Notizie interessanti sulla chiesetta di S. Rocco si trovano in Antonio Alisi, Istria. Città minori, Trieste 1997, 214; Chiara Vigini Conti, "La chiesa di San Rocco e i restauri artistici", Umago viva, 83, 2002, 10; Parentin, Incontri con l'Istria, 24; Fumis, Pagine di storia umaghese, 39. La chiesa di S. Rocco si presenta nella sua forma rettangolare (9,20 x 5,60 m.), a corsi regolari di pietra bianca disposti simmetricamente; ha un'aula unica dominata dall'altare, con tre nicchie di pietra bianca, che ha sostituito a fine Ottocento quello ligneo antico finemente lavorato e lumeggiato a foglia d'oro. Nelle nicchie trovano posto le statue policrome di S. Rocco (1,50 m.), S. Cristoforo (1,20 m.) e S. Sebastiano (1,30 m.) risalenti al XVII secolo; ai lati dell'altare, appoggiate su mensole, sono poste le statue di S. Antonio (0,90 m.) e S. Pellegrino col modello della chiesa parrocchiale in mano, quest'ultima risalente al XV secolo. Il soffitto è decorato con un rivestimento ligneo sul quale Domenico Martinelli dipinse, nel 1788, un S. Rocco in atteggiamento di orante; due dei quattro angeli dipinti agli angoli tengono un nastro ondulante recante le iscrizioni: Hic est qui multum orat pro populo et universa civitate e Peregrinus a nobis susceptus est ut pater noster vocaretur et adoraretur ab omnibus (Narcisa Bolšec-Ferri - Branka Milošević, "Baština Umaga i okolice - Il patrimonio di Umago e dintorni”, Monografia storica Umag-Umago, Umago 2012, 77-78.

39 Andrea Benedetti, Umago d'Istria nei secoli, II, Trieste 1975, 167. Anche Cigui, "Contributo all'araldica di Umago", 255.

40 Flavio Blondio, "Italiae Illustratae undecima regio Histria", AT, II, 1830, 21.

41 Giovan Battista Goineo, "De situ Istriae", $A T$, II, 1830, 64.

42 Ljerka Šimunković, Dalmacija godine gospodnje 1553: Putopis po Istri, Dalmaciji i Mletačkoj Albaniji 1553 godine. Zapisao Zan Battista Giustinian [Dalmazia anno del signore 1553: Itinerario per l’Istria, la Dalmazia e l'Albania veneziana scritto da Zan Battista Giustinian], Spalato 2011, 149. 
cesco Basadonna sottolineò che Umago e le altre terre e città marittime istriane, seppure dotate di porti, erano "quasi spopolate, ripiene di rovine, d'immonditie, d'aria morbosa, poco differenti l'una dall'altra nel numero degli abitanti”. Secondo il provveditore, alla purificazione dell'aria avrebbe giovato "instituire qualche ordine per far tener nette quelle Città e Terre dalle rovine et immonditie, et medesimamente quelle radunanze d'acque piovane, che s'usano per mancamento d'acque vive, che vengono nel paese chiamati laghi, et che s'adoprano in quella provintia in tutte le cose necessarie, se bene per le immonditie sono corrotte et putrefatte" ${ }^{43}$ Fu il successore del Basadonna, Giulio Contarini, che ordinò la bonifica di alcuni stagni a Parenzo e a Umago, “i quali per l'acqua corrotta rendeano gravezza all'aria”, e l'asporto delle immondizie dalle strade e dal territorio. ${ }^{44}$ Tuttavia, alla fine del XVII secolo le condizioni igienico - sanitarie della località continuarono ad essere pessime se nelle sue Memorie sacre e profane dell'Istria (1681) il medico e corografo Prospero Petronio affermò che la cittadina, un tempo molto abitata "hora per l'aria cattiva, per li venti ostro, siroco, garbin che l'offendono resta com'annichilito ripieno d'horrore. La gente come tanti cadaveri spirati, tumida, giallastra, non poco perciò compassionata dal sig. Abb. Grillo allorché di passaggio pose à terra, ma con prontezza ritirossi il piede". ${ }^{45}$

Anche il rifornimento di acqua potabile per le necessità della popolazione rappresentava al tempo un problema di non facile soluzione. Per sopperire alla penuria d'acqua potabile, visto che l'Istria nel primo quarto del XVII secolo disponeva solamente di "quatro piccoli Fiumi, un torrente, et in diversi lochi vi sono alcune Fontane, ma del resto patisce in estremo d'acque", ${ }^{46}$ il Basadonna auspicò la costruzione di cisterne nelle località che ne erano prive poiché tali opere, a fronte di una spesa tutto sommato contenuta, avrebbero determinato "se non ottimi effetti per la sanità de' corpi in particolare, et rispetto agli altri grandissimi beneficij, che convengono esser noti”. ${ }^{47}$ Appena nel 1678, dopo che per secoli il castello di Umago "sitibondo correva per avanti all'acque fetidi del Lago vicino atto solo a trar la

\footnotetext{
43 "Relazioni di provveditori veneti in Istria", AMSI, V, 1889, 94.

44. Idem, 110.

45 Petronio, Delle memorie, 202.

46 "Relazioni di provveditori veneti in Istria", 93.

47 Idem, 95 .
} 
sete alle sanguisuche che vi stagnano”, ${ }^{4}$ il podestà e capitano di Capodistria Angelo Morosini ordinò la costruzione di una cisterna "bella e capace di sicuro refrigerio a quei abitanti” ${ }^{49}$

La costruzione della cisterna pubblica, tuttavia, per quanto contribuisse ad alleviare l'atavica sete della popolazione, non risolse del tutto il problema dell'approvvigionamento idrico della città, che nei decenni successivi continuò ad essere la preoccupazione più sentita dagli abitanti. Nel XVIII secolo la situazione tornò ad essere talmente drammatica che il podestà e capitano di Capodistria Antonio Dolfin, dopo un sopralluogo compiuto nel 1777 a Umago e Cittanova, parlò di luoghi “totalmente rovinosi nel loro Fabricato e spopolati mentre superflua e vana si renderebbe ogni attenzione onde rimeterli, bastando soltanto, che la Publica carità s'impegni a provvederli di acque a suffragio di quelle ristrette popolazioni, specialmente Umago che si ripara col mezzo di una stagnante comune alli Uomini et agli animali”. ${ }^{50}$

\section{Il ripopolamento del territorio}

Come abbiamo potuto appurare dalle corografie, dalle relazioni dei provveditori veneti in Istria e dei podestà e capitani di Capodistria, nei secoli XVI e XVII Umago e il suo territorio erano quasi del tutto deserti, circostanza che spinse la Serenissima ad intraprendere un'intensa azione colonizzatrice nella speranza di ripopolare le campagne abbandonate e scongiurarne in tal modo l'inevitabile tracollo economico.

I primi tentativi veneziani di ripopolare i territori istriani stanziandovi nuove genti risalivano tuttavia al 1376 quando, "pro bono et habitatione terrarum et locorum nostrarum Istriae", la Dominante decise di liberare per cinque anni da ogni tributo e prestazione personale e reale tutti coloro che entro un anno si fossero insediati in qualsiasi parte della provincia istriana. ${ }^{51}$ Tali disposizioni, tuttavia, come del resto tutte le altre di contenuto analogo emanate nel corso dei secoli XIV e XV, avevano per lo più un carattere contingente e rientravano in quelle norme consuetudinarie "che regolavano la sistemazione degli stranieri o delle genti del contado riunendoli nella vicinia, o mirarono localmente a vincolare alla terra le plebi rustiche con delle

\footnotetext{
48 Petronio, Delle memorie, 204.

49 "Relazioni di provveditori veneti in Istria", 135.

50 Benedetti, Umago d'Istria nei secoli, 126.

51 Carlo de Franceschi, L'Istria. Note storiche, Parenzo 1879, 208.
} 
misure di liberalizzazione commerciale per limitarne l'emigrazione, provocata spesso in quei tempi dall'inasprimento delle decime e dalle restrizioni poste ai traffici da un regime quasi permanente di guerra o di emergenza sanitaria”. ${ }^{52}$

In ogni caso, i provvedimenti messi in atto da Venezia decretarono l'afflusso verso la penisola istriana di genti etnicamente eterogenee provenienti da aree geografiche differenti che, non di rado, incontrarono problemi ad acclimatarsi in un ambiente così ostile e a volte così diverso da quello che avevano lasciato. Nella seconda metà del XV secolo, d'altronde, furono proprio le epidemie, le privazioni e le desolazioni recate dalle guerre a determinare il fallimento di un tentativo di colonizzazione con sudditi veneti del Padovano, del Trevisano e del Friuli, i quali trovarono soprattutto grandi difficoltà di adattamento alle terre istriane poverissime d'acqua che richiedevano accorgimenti e metodi di lavoro ben diversi da quelli delle fertili pianure venete e friulane ed enormi fatiche per ricavare un po' di grano, d'olio e di vino. ${ }^{53}$ La Serenissima cercò allora di dar rifugio nelle plaghe deserte dell'Istria alle popolazioni slave, greche e albanesi cacciate dalle regioni balcaniche dai Turchi nel pieno della loro forza espansiva, e già nel 1463 Salvore, desolata dalla peste, venne ripopolata con una colonia di immigrati slavi. ${ }^{54}$

Ben altro significato assunse, a partire dal 1520, l'avvio della cosiddetta colonizzazione organizzata, un processo che si protrasse per circa un secolo e mezzo, nel corso del quale l'incidenza delle guerre e delle pestilenze sulla popolazione giocarono probabilmente un ruolo cruciale nel determinare fasi di stasi o di accelerazione del fenomeno migratorio. ${ }^{55}$ Questa seconda colonizzazione si diversificò nettamente dalla prima, sia per l'intensità del flusso migratorio, che determinò un numero sempre crescente di insediamenti, sia per le ingenti spese sostenute dalla Serenissima nell'organizzare lo spostamento dei coloni dalle regioni di origine alle campagne istriane, fondi che

52 Giulio Cervani - Ettore de Franceschi, "Fattori di spopolamento nell'Istria veneta nei secoli XVI e XVII", ACRSR, IV, 1973, 94.

53 Ernesto Sestan, Venezia Giulia. Lineamenti di una storia etnica e culturale, Bari 1965, 54.

54. Guerrino Girolamo Corbanese, Il Friuli, Trieste e l'Istria dalla preistoria alla caduta del patriarcato d'Aquileia (in seguito: Il Friul), Udine 1983, 320. Per una ricostruzione cronologica del fenomeno migratorio si veda Angelo Marsich, "Gli Slavi in Istria, quando e come vennero", $A T$, XIII, 1887, 411-429.

55 Egidio Ivetic, La popolazione dell'Istria nell'età moderna. Lineamenti evolutivi, Trieste-Rovigno 1997, 170. Per una più attenta analisi dei vari fattori che determinarono il processo di colonizzazione si veda in particolare i lavori di Miroslav Bertoša, Mletačka Istra u XVI $i$ XVII stoljeću [L’Istria veneta nel XVI e XVII secolo], Pola 1986, 46-52 e Istra: doba Venecije (XVI. - XVIII. stoljé́e) [Istria: l'epoca veneziana, XVIXVIII secolo], Pola 1995, 49-55. 
furono in parte gestiti dal Capitanato di Raspo cui spettò l'esclusiva competenza giurisdizionale sui nuovi abitanti. ${ }^{56}$

Nel secoli XVI e XVII anche l'agro umaghese, come del resto gli altri territori dell'Istria, fu interessato dall'importazione di popolazioni allogene nella speranza di risollevarlo dalle vicissitudini che l'avevano colpito. Il senato veneto, in data 13 maggio 1539, aveva decretato che "essendo ritornate alla divotione del nostro Stato due mille Case de Murlachi, le quali viveano all'obbedienza del Turco, ed ora vogliono abitare nei nostri luoghi dell'Istria, (...) non siano tenuti a pagar tassa alcuna per i pascoli; che possano fabbricare fuori della città e castelli dei Casoni per meglio attendere alla pastorizia ed agricoltura, e che per due anni siano esenti dalle gravezze di carriaggi, o di altro che si sogliono imporre ai sudditi nostri” ${ }^{57}$ I Murlachi citati nel decreto, più che a definire etnograficamente un popolo, rappresentavano in realtà un miscuglio di genti sia rumene che slave abitanti le aree montuose della Bosnia, dalle quali, sotto l'incalzare dell'esercito ottomano, si erano riversate nell'area dalmata; il termine, all'epoca, veniva adoperato per indicare generalmente i pastori venuti dai confini turchi. ${ }^{58}$

La Serenissima conosceva molto bene questa popolazione in quanto vari gruppi di morlacchi si erano stabiliti già da tempo nella penisola, per cui non deve stupire che ne avesse approvato il trasferimento. La decisione del senato veneto di assecondare tali genti, desiderose di venire ad abitare la penisola, ebbe un immediato riscontro e, negli anni 1540-41, con morlacchi e dalmati furono ripopolate le campagne abbandonate nei pressi di Umago. ${ }^{59}$ Le terre che queste genti venivano a colonizzare erano aride e poco produttive, molto simili a quelle che avevano abbandonato, terre che per essere lavorate richiedevano una grande capacità di adattamento e una notevole forza fisica. Queste genti, differentemente da quelle provenienti dalla vicina penisola italiana che non erano riuscite ad assuefarsi alla nuova realtà, sembravano invece possedere tutti i requisiti necessari per una rapida integrazione, a partire dalla robusta costituzione fisica che permetteva loro di "far ogni fatica et masime nella galìa” e di lavorare i terreni concessi da Venezia

56 Egidio Ivetic, "Finanza pubblica e sistema fiscale nell'Istria veneta del Sei-Settecento", ACRSR, XXVIII, 1998, 152-153.

57 "Senato Mare. Cose dell'Istria", AMSI, IX, 1893, 122. Vedi anche Bernardo Schiavuzzi, "Cenni storici sull'etnografia dell'Istria" (in seguito: "Cenni storici"), AMSI, XVIII, 1902, 95.

58 Oscar Randi, "I Morlacchi“, Rivista dalmatica, XI, 1929, 27. Sul problema dei morlacchi rimandiamo al lavoro di Egidio Ivetic, L'Istria moderna 1500-1797. Una regione confine, Verona 2010, 138-140.

59 Corbanese, Il Friuli, 322; Schiavuzzi, "Cenni storici”, 96. Cfr. Miroslav Bertoša, "L’Istria veneta nel Cinquecento e nel Seicento", ACRSR, VII, 1976-1977, 147. 
"li quali sono sterilissimi, ma tutto fanno a forza di grandissima fatica per esser montagne e sasso". ${ }^{60}$

Che si trattasse di insediamenti di una certa consistenza, che colmavano il vuoto demografico causato dalle frequenti epidemie e guerre salvando le campagne dallo spopolamento totale, è dimostrato dal fatto che le 25 famiglie morlacche documentate nel 1554 nel comprensorio comunale, circa 125 individui, rappresentavano il 17,5\% della popolazione complessiva che assommava a 715 anime, una percentuale che era quasi analoga a quella della popolazione morlacca insediata in Istria nel ventennio 1534 - 1554 (17\%). ${ }^{61}$ A questi arrivi, nei decenni successivi, se ne aggiunsero ben presto altri: nel 1581 il Capitano di Raspo Alessandro Zorzi informò il governo veneto della presenza di 120 morlacchi nell'agro umaghese ${ }^{62}$ e, nel 1599, altri habitanti novi si insediarono nelle campagne tra Umago e Buie ${ }^{63}$. Un discorso a parte merita l'emigrazione nell'Umaghese, e più in generale nella penisola, dei cosiddetti Cargnelli, genti provenienti dalle regioni montuose della Carnia che sin dal basso medioevo si erano stanziate in Istria e il cui flusso, a partire dal XVI secolo, assunse proporzioni sempre più rilevanti. ${ }^{64}$ Tale emigrazione, che ebbe un carattere prettamente individuale in quanto non fu gestita dalla Dominante, portò i carnici ad insediarsi prevalentemente nelle aree interne della penisola dove, in un'area prevalentemente agricola e dedita all'allevamento, supplirono alla totale mancanza di manodopera artigianale.

L'insediamento dei coloni si effettuava mediante investitura, per cui venivano concessi loro fondi e casali e godevano, per vent'anni, esenzione da ogni gravame personale e reale, affrancati dalla giurisdizione dei rispettivi comuni di residenza. Le profonde differenze culturali e religiose tra originari e forestieri furono tuttavia frequentemente oggetto di una forte

60 Giovanni Netto, "La terraferma veneziana nel 1555”, Archivio veneto, CXXVI, 1986, 33.

61 Miroslav Bertoša, "Istarski fragment itinerara mletačkih sindika iz 1554 godine" [Il frammento istriano dell'itinerario dei sindici veneziani del 1554], VHARP, XVII, 1972, 42-43; idem, Mletačka Istra u XVI $i$ XVII stoljeću, 311 ; idem, Istarsko vrïeme prošlo [L'Istria dei tempi passati], Pola 1978, 203.

62 "Relazioni di provveditori veneti in Istria”, AMSI, VI, 1890, 96.

63 “Senato Mare. Cose dell'Istria”, AMSI, XII, 1896, 84. “Avendo il podesta' di Umago e quello di Buie pronunciate sentenze contro Martino Villanovich e Paolo Glamovich, ed essendo questi due del numero dei nuovi abitanti, si commette alle cariche suddette d'inviare tosto i processi relativi al Capitano di Raspo, a cui spettano tali giudizi". E ancora il 28 agosto 1603 "avendo il podestà di Umago bandito per sei anni da questa terra Martino Villanovich, che per essere nuovo abitante dipende dalla giurisdizione di Raspo, ed avendogli ancora mutato il bando in pena pecuniaria, gli si ordina la restituzione del denaro ricevuto intimandogli che non s'intrometta più oltre nelle cause relative ai nuovi abitanti” (p. 103). Cfr. Benedetti, Umago d'Istria nei secoli, 118-121.

64 Dean Brhan, “In partibus Carsi et Istriae: l'emigrazione dalla Carnia verso l'Istria (XVI-XIX secolo)”, ACRSR, XXXIV, 2004, 474. 
conflittualità, sulla quale incisero altresì la ripartizione dei beni sfruttati collettivamente, le esenzioni fiscali e i privilegi giudiziari di cui godevano i colonizzatori. ${ }^{65}$ I nuovi abitanti, infatti, si erano trasferiti in Istria portando con sé un codice morale e comportamentale non sempre compatibile con la tradizione locale e con la legislazione veneziana. A creare frequenti problemi fu soprattutto il ricorso alla violenza privata, un sistema di regolazione dei contenziosi all'interno delle singole comunità ampiamente diffuso tra le popolazioni morlacche, fermamente condannato dalla Repubblica di Venezia sia con dure sanzioni sia mostrando come convenisse agli stessi interessati ricorrere ai tribunali marciani, dove potevano veder ascoltate le loro richieste. ${ }^{66}$

Molte fonti descrivevano queste persone come rissose, violente e di pessima qualità. Nella relazione del Capitano di Raspo Giacomo Renier (1594) i morlacchi vennero raffigurati come "barbara gente, inutile per la dappocaggine e crepula e fuga della fatica al remo, alla spada, alla campagna, solo nota per ubbriacarsi, stare alle strade ed assassinare i popoli, caione principale per li loro infiniti furti di animali, ed altri danni che fanno" ${ }^{67}$, mentre il podestà e capitano di Capodistria Marin Gradenigo riferiva, nel 1608, le lamentele delle genti autoctone per "il mal trattamento che ricevono dalli novi habitanti li quali, oltra che si fanno investir di molti terreni che non gli aspettano scacciando li habitanti vecchi che li possedono, li fanno anco molti affronti et insolenze, perché non potendo essi novi habitanti esser chiamati in giuditio né civil né criminalmente se non inanzi l'Ill.mo S.r Capitano di Raspo loro giudice delegato, (...) essi novi habitanti s'inanimiscono e prendono fomento di strussiarli e di far nelli territorij dove habitano molte insolenze a danno e pregiudizio non solamente delli vecchi habitanti (...), ma con poco rispetto anco degli istessi Rettori, nel distretto de’ quali vengono ad habitare”68. Anche le trenta famiglie trasportate nel 1603 a Giuba e Seghetto dal capitano Vincenzo Chiucchel da Sebenico ${ }^{69}$

65 Giuliano Veronese, "L’immigrazione nell'Istria veneta tra ‘500 e ‘600: problemi giurisdizionali, contese tra comunità, conflitti etnici tra originari e forestieri”, Acta Histriae, III, 1994, 183.

66 Claudio Povolo, "Rappresentazioni dell'onore nel discorso processuale (da una vicenda degli inizi del Seicento)", Acta Histriae, X, 2000, 513-534.

67 De Franceschi, L'Istria. Note storiche, 369.

68 "Relazioni di provveditori veneti in Istria”, AMSI, VII, 1891, 149.

69 Schiavuzzi, “Cenni storici”, 368. Il governo veneto remunerava il Chiucchel con sei ducati al mese vita natural durante, e prometteva di dare in prestito trenta ducati per famiglia perché provvedessero agli animali necessari. Il terreno concesso alle trenta famiglie aveva una circonferenza di circa quattro miglia. Nel 1623 il Chiucchel vendette la tenuta ai Valier di Venezia e acquistata, un secolo dopo, dalla famiglia de Franceschi. Cfr. "Senato Mare. Cose dell’Istria”, AMSI, XII, 1896, 101. 
si erano rese colpevoli di mortali ferimenti a danno dei vecchi abitanti, di arrembaggio a una nave da carico sul Quieto e di altri delitti. Lapidario fu il giudizio espresso nel 1625 dal provveditore veneto Francesco Basadonna sui nuovi venuti: "I nuovissimi, terza fonte di abitanti nuovi, essendo poverissimi e miserabili, sono per la maggior parte ladri, fanno danni notabili agli abitanti vecchi negli animali e nei raccolti". ${ }^{70}$

Nel 1612 otto famiglie albanesi provenienti da Cugnia si stanziarono a Petrovia. Il 12 gennaio di quell'anno il Capitano di Raspo Pietro Bondumier aveva accolto la supplicazione di Nico Babich, Pietro Nico e dei loro compagni i quali ottenevano settecento campi “inculti spinosi e sassosi nelle pertinenze di Umago nella contrada di Malladelle con l'obbligo di ridur quelli a perfetta coltura, e li non habili a coltura risseruar per pascolo delli Anemalli". ${ }^{71}$ Lassegnazione di un numero così cospicuo di terreni da parte veneta non deve sorprendere in quanto le aree abbandonate e incolte del territorio umaghese, evidenziate dal perito et ingegnero Camillo Bergami nel 1613, ammontavano alla misura padovana a campi 187, quarte 3, tavole 78, corrispondenti a 72,5 ettari. ${ }^{72}$ Altri albanesi presero dimora a Petrovia nel 1627: ai nuovi abitanti venne concesso dal Senato, nel 1640, di fabbricarsi una semplice cappelletta ${ }^{73}$.

Un nuovo contingente di morlacchi venne collocato dal Capitano di Raspo nel 1647 nei territori di Pola, S. Lorenzo e Umago. Il 17 agosto il Senato registrò un imbarco di 1.500 uomini e 3.450 donne e bambini con 5.000 animali grossi e 65.000 minuti, uno dei più grossi trasferimenti nella lunga storia della ripopolazione dell’Istria. I morlacchi che raggiunsero l'Istria furono sistemati "non lungo le marine, ma entro terra ove maggiori sono le comodità". Non solo ai nuovi venuti furono accordati terreni incolti

70 Bernardo Benussi, L'Istria nei suoi due millenni di storia, Venezia-Rovigno 1997, 344.

71 Archivi Capitolare di Umago (in seguito: ACU), Scritture Spettanti al Gius Episcopale e Capitolare d'Umago uniti l'anno 1706. Li abitanti, manoscritto, XVII-XVIII secolo.

72 Miroslav Bertoša, "I catastici di Umago e di Cittanova (1613-1614). La modesta realizzazione di un grandioso disegno nell'Istria veneta (XVI-XVII sec.)”, ACRSR, IX, 1979, 430. Che tale situazione perdurasse ancora nel Settecento lo confermò il canonico della Collegiata di Umago Francesco Roselli (1739-1798): "la maggior parte delle Terre soggette alla mia Parrochia sono incolte, o come si dice in Baredo, atteso la scarsezza della gente profuga, e disanimata in lavorar i Terreni che sono in gran parte sassosi, o spinosi, e soprattutto soggetti alla siccità, come ovunque abbastanza consta esser di tutta questa Provincia infelice sempre, e desolata per la qualità de Terreni arridi, e per la costituzione naturale del clima nell'estate insalubre; cosicché ogni venti campi di terra se ne mette a coltura per uno circa, che si sceglie tra i meno cattivi, e che d'ordinario dai Proprietarj si fà lavorare a proprio conto con Opere di Lavoratori giornalieri, che alla giornata vengono spesati, e pagati. Tra questi beni è compreso il Luogo di Sipar soggetto pure alla mia Pieve, e così s'intende delle Terre di tutto il Territorio del più e del meno consimili” (Archivio Benedetti, Stampa dell'L.L. C.C. Conti Rotta al Taglio, manoscritto, XVII - XVIII secolo).

73 "Senato Mare. Cose dell'Istria", AMSI, XV, 1899, 21. 
e beni pubblici, ma anche la possibilità di accasarsi quali coloni su terreni altrui. $^{74}$ Va però sottolineato che la Repubblica di Venezia, propensa a favorire in ogni modo l'arrivo di nuove popolazioni, poneva l'accettazione della religione cattolica quale condizione essenziale per essere accolti nei territori veneti. Il Senato, infatti, sin dal 1651, aveva provveduto a far pubblicare a Umago un proclama che imponeva l'allontanamento dall'Istria di chi non si fosse adattato a professare la nuova religione. ${ }^{75}$ Dopo l'insediamento degli albanesi nel 1627, le autorità incaricarono il pubblico perticatore Giacomo Viuchio della misurazione dei terreni ancora disponibili a eventuali assegnazioni. Dalla relazione stesa nel 1633 veniamo a sapere che "furono perticati campi 783 Tauole 641 e di questi venduti campi 185 Tauole 638 nel sito della Villa intitolata Petrognia et suoi Horti”; rimanevano ancora liberi "per ragioni d'Investitura alli noui Habitanti in detta Villa campi cinquecento nouanta otto Tauole 3 cioè 598 T.le $3{ }^{7}{ }^{76}$

Gli uomini di Petrovia, per i loro animali, usufruivano del Bosco di Pizzudo, del Bosco Grando e della Punta di Rosazzo. Il 6 maggio 1693 Catterina Bernardo, consorte del sig. Pietro Valier, affittava a Mattio Jurzan, Mattio Usco, Zuanne Marcouich, Marina Vitanouichia e Antonia figlia del fu Mattio Jon "tutti sud.ti della Villa di Habitantia, per li loro Animali il pascolo del Bosco grande (...) e il transito per rosazo al mare e ciò per lire cento all'anno da pagarsi in tre rate principiando la prima il primo di Maggio".77

Nonostante fossero ormai scaduti i vent'anni di esenzione da ogni gravame personale e reale, i nuovi abitanti si rifiutavano di versare il dovuto alla podesteria e capitolo umaghesi e al vescovo di Trieste. "Li suditi del teritorio d'Umago, Carse e Materada” - osservava il cancelliere Michiel Rosello nel 1706 - "paghano tutti il Teradico ed erbaticij et altre decime solitte a q.ta Comunità di Umago come il Trentese e quartese alla Mensa Episcopalle e Capitollo di questa Terra e questo per Antichissima consuetudine uiene contribuitto da tutti come sopra foriche quelli del Comun di Petrouia che per hauer certa Investitura priuata del 1612 non pagano Cosa alcuna”. ${ }^{78}$

Nei primi anni del Settecento le autorità venete, stanche delle ripetute inadempienze, intimarono a Mattio Puisa, zuppano di Petrovia, di conse-

\footnotetext{
74 Idem, XVIII, 1902, 241. Cfr. Schiavuzzi, “Cenni storici”, 233.

75 Benedetti, Umago d'Istria nei secoli, 125.

76 ACU, Scritture Spettanti al Gius Episcopale, documento 18/12/1633.

77 Idem, documento 6/5/1693.

78 Idem, documento $28 / 6 / 1706$.
} 
gnare alla cancelleria di Umago una lista di tutti i capifamiglia in possesso di case o terre "contro i termini delli beni assegnategli nella investitura 1612 fatta alli Abitanti all'hora uenuti dall'Albania, affinchè deuano presto nel fontaco di Umago il Trentesimo e quarantesimo de grani pretesi da essa mensa". ${ }^{79}$ I nominativi dei capi di casa tutti erano: Sig.r Gio. Marquiz e famiglia, Stane Radesigi, Mattio Giorizan, Mattio Poiza, Mattio Vocouiz, Zuane Sossa, Capo Luca Giorgiouigi, Mattio Giorgiouigi, Gasparo Codam, Zorzi Sestaco, Antonio Usco o Vocassino.

\section{Conclusione}

Come si evince da quanto esposto, i secoli XVI e XVII furono cruciali per la storia di Umago. Si trattò in realtà di un'epoca contraddistinta da forti chiaroscuri, nel corso della quale le premesse per un decollo socio-economico e demografico andarono inevitabilmente a scontrarsi con il generale degrado ambientale e igienico-sanitario della località che, malgrado le notevoli risorse del territorio e il trapianto di popolazioni, ebbe conseguenze deleterie sia sul piano della crescita economica sia su quello demografico. La società umaghese dell'epoca era ancora tipicamente agricola, ma al suo interno si stavano lentamente affermando attività quali l'artigianato, la marineria e la pesca destinate ad affermarsi definitivamente nei secoli successivi.

\footnotetext{
Riassunto

Umago, che aveva vissuto nei primi secoli dopo il Mille un discreto periodo di prosperità economica e demografia che ne aveva decretato l'espansione sulla terraferma, nei secoli successivi fu inserita nel novero delle zone insalubri della provincia, e nonostante nei secoli XVI e XVII vi fossero tutti i presupposti per un seppur modesto decollo economico e demografico, questo non avvenne a causa delle pessime condizioni igieniche e sanitarie che rallentarono la crescita della popolazione, e di riflesso, quella economica. Il depauperamento demografico della città e del territorio fu contrastato dall'intensa azione colonizzatrice della Serenissima, che vi trapiantò popolazioni provenienti dall'area balcanica e non solo nella speranza di ripopolare le campagne abbandonate e scongiurarne in tal modo il tracollo economico. Iniziò così un processo, durato un secolo e mezzo, che colmò il vuoto demografico causato dalle frequenti epidemie e guerre, ma alterò nel contempo la struttura etnica del territorio.
}

79 Idem, documento 20/7/1706. 


\section{Neki aspekti socio-ekonomskoga života u Umagu u XVI. i XVII. stoljeću}

\section{Sažetak}

Za povijest Umaga presudnima su bili XVI. i XVII. stoljeće. Mjesto koje je u prvim stoljećima nakon tisućite godine proživjelo razdoblje umjerenoga gospodarskoga i demografskoga razvitka koji mu je odredio širenje u zaleđe, u sljedećim je stoljećima doživjelo uvrštavanje u red nezdravih područja provincije. Usprkos tome što su u XVI. i XVII. stoljeću postojale sve pretpostavke za, premda skroman, gospodarski i demografski pomak, to se nije dogodilo zbog izuzetno loših higijenskih i sanitarnih uvjeta koji su usporavali prirast stanovništva a, neizravno, i gospodarski razvoj. Demografskom osipanju grada i okolice suprotstavljalo se intenzivno kolonizatorsko djelovanje Mletačke Republike, koja je doseljavala stanovništvo porijeklom s balkanskih prostora i to ne samo u nadi da opet naseli napuštena sela i tako otkloni gospodarsko propadanje. Počeo je jednoipolstoljetni proces koji je popunio demografsku prazninu uzrokovanu čestim epidemijama i ratovima te istovremeno izmijenio etničku strukturu cijeloga područja.

\section{Some aspects of socio-economic life in Umag in $16^{\text {th }}$ and $17^{\text {th }}$ century}

\section{Summary}

$16^{\text {th }}$ and $17^{\text {th }}$ century were crucial for the history of Umag. A city which had a moderate economic and demographic development in the first centuries of the $2^{\text {nd }}$ millennium A.D., and consequently spread to hinterland, was categorized as an unhealthy part of the province in the next centuries. Despite having the prerequisite for, although modest, economic and demographic progress, it did not occur because of bad hygienic and sanitary conditions which slowed the growth of population and with that, indirectly, the economic development. Demographic decrease which affected the city and its surrounding area was in opposition to intensive Venetian colonisation which brought people from the Balkans hoping to populate abandoned villages thus removing the economic decline. With that begun the century and a half long process which filled the demographic void, caused by frequent epidemics and wars, and at the same time it changed the ethnic structure of the entire area. 
